\title{
MAXIMUM AND COMPARISON PRINCIPLES FOR CONVEX FUNCTIONS ON THE HEISENBERG GROUP
}

\author{
CRISTIAN E. GUTIÉRREZ \\ AND \\ ANNAMARIA MONTANARI
}

\section{INTRODUCTION}

The purpose in this paper is to establish pointwise estimates for a class of convex functions on the Heisenberg group. An integral estimate for classical convex functions in terms of the Monge-Ampère operator det $D^{2} u$ was proved by Aleksandrov, see [3, Theorem 1.4.2]. Such estimate is of great importance in the theory of weak solutions for the Monge-Ampère equation, and its proof revolves around the geometric features of the notion of normal mapping or subdifferential in $\mathbb{R}^{n}$ [3, Definition 1.1.1] which yield in addition the useful comparison principle for Monge-Ampère measures, [3, Theorem 1.4.6].

On the Heisenberg group, and more generally in Carnot groups, several notions of convexity have been introduced and compared in [2] and [4]. The notion of convex function we use in this paper is given in Definition 2.2, and a natural question is if similar comparison and maximum principles hold in this setting. A reason for this question is that those estimates would be useful in the study of solutions for nondivergence equations of the form $a_{i j} X_{i} X_{j}$ where $a_{i j}$ is a uniformly elliptic measurable matrix and $X_{i}$ are the Heisenberg vector fields. The difficulty for this study is the doubtful existence of a notion of normal mapping in $\mathbb{H}^{n}$ suitable to establish maximum and comparison principles.

In this paper we address this question and follow a route different from the one described above for convex functions, and in particular, we do not use any notion of normal mapping. This approach was recently used by Trudinger and Wang to study Hessian equations [6]. Our integral estimates are in terms of the following Monge-Ampère type

November 13, 2002.

The results in this paper and the ideas of their proofs have been presented in the following talks: Analysis Seminar, Temple U., October 2002; Fabes-Chiarenza Lectures at Siracusa, December 2002; Pan-American Conference, Santiago de Chile, January 2003, Analysis Seminar, U. of Bologna, March 2003, and Analysis Seminar, U. Texas at Austin, March 2003.

The first author was partially supported by NSF grant DMS-0070648, and thanks the University of Bologna for its support and hospitality in his several visits to carry out this project. The second author thanks Temple University for the hospitality during her visit during March-April 2001 when the key Theorem 3.1 was proved. 
operator: $\operatorname{det} \mathcal{H}(u)+12\left(u_{t}\right)^{2}$, see Definition 2.1. We first establish by means of integration by parts a comparison principle for smooth functions, Theorem 3.1, and then extend this principle to "cones" Theorem 4.7. This together with the geometry in $\mathbb{H}^{n}$ leads by iteration to the maximum principle Theorem 5.5. We next estimate the oscillation of $\mathcal{H}_{-}$ convex functions Proposition 6.2 that permits to extend our definition of Monge-Ampère measure to continuous $\mathcal{H}$-convex functions and obtain a general comparison principle Theorem 6.7.

The paper is organized as follows. Section 2 contains preliminaries about $\mathbb{H}^{n}$ and the definitions of $\mathcal{H}$-convexity. In Section 3 we prove the comparison principle for $C^{2}$ functions. Section 4 contains the proof that "cones" agreeing with $\mathcal{H}$-convex functions $u$ on the boundary are above $u$ inside, and the comparison principle for cones Theorem 4.7. In Section 5 we prove a maximum principle similar to Aleksandrov's estimate aforementioned. Finally, Section 6 contains the oscillation estimates and the construction of the analogue of Monge-Ampère measures for $\mathcal{H}$-convex functions.

2. Preliminaries and $\mathcal{H}$-convexity

Let $u=u(x, y, t) ; z=(x, y, t)$, and $X=\partial_{x}+2 y \partial_{t}, Y=\partial_{y}-2 x \partial_{t}$. We have $[X, Y]=X Y-$ $Y X=-4 \partial_{t}$. If $\xi_{0}=\left(x_{0}, y_{0}, t_{0}\right)$ and $\xi=(x, y, t)$, then the non-commutative multiplication law in $\mathbb{H}^{1}$ is given by

$$
\xi_{0} \circ \xi=\left(x_{0}+x, y_{0}+y, t_{0}+t+2\left(x y_{0}-y x_{0}\right)\right),
$$

and we have $\xi^{-1}=-\xi$, and $\left(\xi_{0} \circ \xi\right)^{-1}=\xi^{-1} \circ \xi_{0}^{-1}$. The gauge in $\mathbb{H}^{1}$ is

$$
\rho(\xi)=\left(\left(x^{2}+y^{2}\right)^{2}+t^{2}\right)^{1 / 4},
$$

and the distance

$$
d\left(\xi, \xi_{0}\right)=\rho\left(\xi_{0}^{-1} \circ \xi\right)
$$

We have

$$
d\left(\xi, \xi_{0}\right) \leq d(\xi, \zeta)+d\left(\zeta, \xi_{0}\right)
$$

for every $\xi, \xi_{0}, \zeta \in \mathbb{H}^{1}$. Given $\lambda>0$ we consider the dilations

$$
\delta_{\lambda}(\xi)=\left(\lambda x, \lambda y, \lambda^{2} t\right) .
$$

Then

$$
d\left(\delta_{\lambda} \xi, \delta_{\lambda} \xi_{0}\right)=\lambda d\left(\xi, \xi_{0}\right) .
$$

For more details about $\mathbb{H}^{n}$ see [5, Chapters XII and XIII].

2.1. $\mathcal{H}$-convexity. Let $\xi_{0}=\left(x_{o}, y_{o}, t_{o}\right), \zeta=(x, y, t)$ and

$$
g(\zeta)=f\left(\xi_{o} \circ \zeta\right)
$$

We have

$$
\partial_{x} g(0)=X f\left(\xi_{o}\right), \quad \partial_{y} g(0)=Y f\left(\xi_{o}\right), \quad \partial_{t} g(0)=\partial_{t} f\left(\xi_{o}\right),
$$

and

$$
\begin{array}{lll}
\partial_{x x} g(0)=\left(X^{2} f\right)\left(\xi_{0}\right), & \partial_{x y} g(0)=(Y X f)\left(\xi_{0}\right)-2 \partial_{t} f\left(\xi_{o}\right), & \partial_{x t} g(0)=\partial_{t x} f\left(\xi_{0}\right)+2 y_{0} \partial_{t t} f\left(\xi_{o}\right), \\
\partial_{y x} g(0)=(X Y f)\left(\xi_{0}\right)+2 \partial_{t} f\left(\xi_{o}\right), & \partial_{y y} g(0)=\left(Y^{2} f\right)\left(\xi_{0}\right), & \partial_{y t} g(0)=\partial_{t y} f\left(\xi_{0}\right)-2 x_{0} \partial_{t t} f\left(\xi_{o}\right),
\end{array}
$$


$\partial_{t x} g(0)=\partial_{t x} f\left(\xi_{0}\right)+2 y_{0} \partial_{t t} f\left(\xi_{o}\right), \quad \partial_{t y} g(0)=\partial_{t y} f\left(\xi_{0}\right)-2 x_{0} \partial_{t t} f\left(\xi_{o}\right), \quad \partial_{t t} g(0)=\partial_{t t} f\left(\xi_{0}\right)$.

Let

$$
A=\left[\begin{array}{ccc}
\left(X^{2} f\right)\left(\xi_{0}\right) & (Y X f)\left(\xi_{0}\right)-2 \partial_{t} f\left(\xi_{o}\right) & \partial_{t x} f\left(\xi_{0}\right)+2 y_{0} \partial_{t t} f\left(\xi_{o}\right) \\
(X Y f)\left(\xi_{0}\right)+2 \partial_{t} f\left(\xi_{o}\right) & \left(Y^{2} f\right)\left(\xi_{0}\right) & \partial_{t y} f\left(\xi_{0}\right)-2 x_{0} \partial_{t t} f\left(\xi_{o}\right) \\
\partial_{t x} f\left(\xi_{0}\right)+2 y_{0} \partial_{t t} f\left(\xi_{o}\right) & \partial_{t y} f\left(\xi_{0}\right)-2 x_{0} \partial_{t t} f\left(\xi_{o}\right) & \partial_{t t} f\left(\xi_{0}\right)
\end{array}\right] .
$$

Then the Taylor polynomial of order two of $g$ is

$$
\begin{aligned}
& f\left(\xi_{o}\right)+\left(X f\left(\xi_{o}\right), Y f\left(y_{0}\right), \partial_{t} f\left(\xi_{0}\right)\right) \cdot \zeta+\frac{1}{2}\langle A \zeta, \zeta\rangle \\
& =f\left(\xi_{o}\right)+\left(X f\left(\xi_{o}\right), Y f\left(y_{0}\right)\right) \cdot(x, y)+\left(X^{2} f\right) x^{2}+(X Y f+Y X f) x y+\left(Y^{2} f\right) y^{2} \\
& \quad+t\left\{f_{t}\left(\xi_{0}\right)+2 f_{t x} x+4 y_{o} f_{t t} x+\left(f_{t x}+f_{t y}\right) y-4 x_{o} f_{t t} y\right\} .
\end{aligned}
$$

That is, if $(x, y, t) \in \Pi_{0}$ then $t=0$ and so on this plane we have

$$
\begin{aligned}
g(\zeta)=f\left(\xi_{o}\right)+\left(X f\left(\xi_{o}\right), Y f\left(y_{0}\right)\right) \cdot(x, y) & \\
& +\left(X^{2} f\right) x^{2}+(X Y f+Y X f) x y+\left(Y^{2} f\right) y^{2}+o\left(x^{2}+y^{2}\right) .
\end{aligned}
$$

Set $B_{R}\left(\xi_{0}\right)=\left\{\xi \in \mathbb{R}^{3}: d\left(\xi, \xi_{0}\right)<R\right\}$. Given $\xi_{0}=\left(x_{0}, y_{0}, t_{0}\right) \in R^{3}$ let

$$
\Pi_{\xi_{0}}=\left\{(x, y, t): t-t_{0}-2\left(x y_{0}-y x_{0}\right)=0\right\} .
$$

That is, $\Pi_{\xi_{0}}$ is the plane generated by the vectors $\left(1,0,2 y_{0}\right),\left(0,1,-2 x_{0}\right)$ and passing through the point $\xi_{0}$. Notice that if $h \in \mathbb{H}^{1}$, then

$$
\xi \in \Pi_{\xi_{0}} \text { if and only if } h \circ \xi \in \Pi_{h \circ \xi_{0}} .
$$

Given $c \in \mathbb{C}$ and $u \in C^{2}(\Omega)$, let

$$
\mathcal{H}_{c}(u)=\left[\begin{array}{cc}
X^{2} u & X Y u+c u_{t} \\
Y X u-c u_{t} & Y^{2} u
\end{array}\right]
$$

and

$$
H_{c}(u)=\operatorname{det}\left[\begin{array}{cc}
X^{2} u & X Y u+c u_{t} \\
Y X u-c u_{t} & Y^{2} u
\end{array}\right]
$$

Definition 2.1. The function $u \in C^{2}(\Omega)$ is $\mathcal{H}$-convex in $\Omega$ if the symmetric matrix

$$
\mathcal{H}(u)=\mathcal{H}_{2}(u)=\left[\begin{array}{cc}
X^{2} u & (X Y u+Y X u) / 2 \\
(X Y u+Y X u) / 2 & Y^{2} u
\end{array}\right]
$$

is positive semidefinite in $\Omega$.

Notice that the matrix $\mathcal{H}_{c}(u)$ is symmetric if and only if $c=2$. Also, if $\left\langle\mathcal{H}_{c}(u) \xi, \xi\right\rangle \geq 0$ for all $\xi \in \mathbb{R}^{2}$ and for some $c$, then this quadratic form is nonnegative for all values of $c \in \mathbb{R}$.

We extend the definition of $\mathcal{H}$-convexity to continuous functions.

Definition 2.2. The function $u \in C(\Omega)$ is $\mathcal{H}$-convex in $\Omega$ if there exists a sequence $u_{k} \in$ $C^{2}(\Omega)$ of $\mathcal{H}$-convex functions in $\Omega$ such that $u_{k} \rightarrow u$ uniformly on compact subsets of $\Omega$.

The following proposition yields equivalent definitions of $\mathcal{H}$-convexity, see [2, Theorem 5.11] for the proof. 
Proposition 2.3. Let $u \in C(\Omega)$ with $\Omega \subset \mathbb{R}^{3}$ open $^{*}$. The following are equivalent:

(1) $u$ is $\mathcal{H}$-convex.

(2) Given $\xi_{0} \in \Omega$

$$
u\left(\xi_{0} \circ \delta_{\lambda}\left(\xi_{0}^{-1} \circ \xi\right)\right) \leq u\left(\xi_{0}\right)+\lambda\left(u(\xi)-u\left(\xi_{0}\right)\right),
$$

for all $\xi \in \Pi_{\xi_{0}}$ and $0 \leq \lambda \leq 1$.

Remark 2.4. From Proposition 2.3(2) we have that if $u$ is convex in the standard sense, then $u$ is $\mathcal{H}$-convex. However, the gauge function $\rho(x, y, t)=\left(\left(x^{2}+y^{2}\right)^{2}+t^{2}\right)^{1 / 4}$ is $\mathcal{H}-$ convex but is not convex in the standard sense, see Proposition 4.5.

\section{Comparison Principle}

We prove the following.

Theorem 3.1. Let $u, v \in C^{2}(\bar{\Omega})$ such that $u+v$ is $\mathcal{H}$-convex in $\Omega$ satisfying $v=u$ on $\partial \Omega$ and $v<u$ in $\Omega$. Then

$$
\int_{\Omega}\left\{\operatorname{det} \mathcal{H}(u)+12\left(\partial_{t} u\right)^{2}\right\} d z \leq \int_{\Omega}\left\{\operatorname{det} \mathcal{H}(v)+12\left(\partial_{t} v\right)^{2}\right\} d z,
$$

and

$$
\int_{\Omega} \operatorname{trace} \mathcal{H}(u) d z \leq \int_{\Omega} \operatorname{trace} \mathcal{H}(v) d z
$$

Proof. If $Z=\alpha_{1} \partial_{x_{1}}+\alpha_{2} \partial_{x_{2}}+\alpha_{3} \partial_{x_{3}}$ is a smooth vector field, then

$$
\int_{\Omega} Z u d x=\int_{\partial \Omega} v_{Z} u d \sigma(x)-\int_{\Omega}\left(\left(\alpha_{1}\right)_{x_{1}}+\left(\alpha_{2}\right)_{x_{2}}+\left(\alpha_{3}\right)_{x_{3}}\right) u d x,
$$

where $v=\left(v_{1}, v_{2}, v_{3}\right)$ is the outer unit normal to $\partial \Omega$ and $v_{Z}=\alpha_{1} v_{1}+\alpha_{2} v_{2}+\alpha_{3} v_{3}$.

Since $v=u$ on $\partial \Omega, v<u$ in $\Omega$ and both functions are smooth up to the boundary, it follows that the normal to $\partial \Omega$ is $v=\frac{D(v-u)}{|D(v-u)|}$, and therefore $v_{X}=\frac{X(v-u)}{|D(v-u)|}$ and $v_{Y}=\frac{Y(v-u)}{|D(v-u)|}$. Set

$$
S(u)=\operatorname{det} \mathcal{H}(u)=X^{2} u Y^{2} u-\left(\left(\frac{X Y+Y X}{2}\right) u\right)^{2} .
$$

We have

$$
\begin{aligned}
& \frac{\partial S(u)}{\partial r_{11}}=Y^{2} u ; \quad \frac{\partial S(u)}{\partial r_{12}}=-\left(\frac{X Y+Y X}{2}\right) u ; \\
& \frac{\partial S(u)}{\partial r_{21}}=-\left(\frac{X Y+Y X}{2}\right) u ; \quad \frac{\partial S(u)}{\partial r_{22}}=X^{2} u .
\end{aligned}
$$

${ }^{*}$ We assume that if $\xi, \xi_{0} \in \Omega$, then $\xi_{0} \circ \delta_{\lambda}\left(\xi_{0}^{-1} \circ \xi\right) \in \Omega$ for $0<\lambda<1$. 
Let $0 \leq s \leq 1$ and $\varphi(s)=S(s u+(1-s) v)$. Then

$$
\begin{aligned}
& \int_{\Omega}\{S(u)-S(v)\} d z \\
& =\int_{0}^{1} \int_{\Omega} \varphi^{\prime}(s) d z d s \\
& =\int_{0}^{1} \int_{\Omega}\left\{\sum_{i, j=1}^{2} \frac{\partial S}{\partial r_{i j}}(v+s(u-v))\left(\frac{X_{i} X_{j}+X_{j} X_{i}}{2}\right)(u-v)\right\} d z d s \\
& =\int_{0}^{1} \int_{\Omega}\left\{\sum_{i, j=1}^{2} \frac{\partial S}{\partial r_{i j}}(v+s(u-v))\left(X_{i} X_{j}\right)(u-v)\right\} d z d s \text { since } S_{i j} \text { is symmetric } \\
& =\int_{0}^{1} \int_{\Omega}\left\{\sum_{i, j=1}^{2} X_{i}\left(\frac{\partial S}{\partial r_{i j}}(v+s(u-v)) X_{j}(u-v)\right)-X_{i}\left(\frac{\partial S}{\partial r_{i j}}(v+s(u-v))\right) X_{j}(u-v)\right\} d z d s \\
& =A-B .
\end{aligned}
$$

We have

$$
\begin{aligned}
& A=\int_{0}^{1} \int_{\Omega} X\left[Y^{2}(v+s(u-v)) X(u-v)\right]-Y\left[\left(\frac{X Y+Y X}{2}\right)(v+s(u-v)) X(u-v)\right] \\
& -X\left[\left(\frac{X Y+Y X}{2}\right)(v+s(u-v)) Y(u-v)\right]+Y\left[X^{2}(v+s(u-v)) Y(u-v)\right] d z d s \\
& =\int_{0}^{1} \int_{\partial \Omega} v_{X} Y^{2}(v+s(u-v)) X(u-v)-v_{Y}\left(\frac{X Y+Y X}{2}\right)(v+s(u-v)) X(u-v) \\
& -v_{X}\left(\frac{X Y+Y X}{2}\right)(v+s(u-v)) Y(u-v)+v_{Y} X^{2}(v+s(u-v)) Y(u-v) d z d s \\
& =-\int_{0}^{1} \int_{\partial \Omega}\left\{Y^{2}(v+s(u-v)) X(u-v)^{2}\right. \\
& +\left(\frac{X Y+Y X}{2}\right)(v+s(u-v)) X(u-v) Y(u-v) \\
& +\left(\frac{X Y+Y X}{2}\right)(v+s(u-v)) Y(u-v) X(u-v) \\
& \left.-X^{2}(v+s(u-v)) Y(u-v)^{2}\right\} \frac{1}{|D(v-u)|} d z d s \\
& =-\int_{0}^{1} \int_{\partial \Omega}\langle\mathcal{H}(v+s(u-v))(X(u-v), Y(u-v)),(X(u-v), Y(u-v))\rangle \frac{1}{|D(v-u)|} d z d s \\
& \leq 0 \text {. }
\end{aligned}
$$


We now calculate $B$

$$
\begin{aligned}
B= & \int_{0}^{1} \int_{\Omega} \sum_{i, j=1}^{2} X_{i}\left(\frac{\partial S}{\partial r_{i j}}(v+s(u-v))\right) X_{j}(u-v) d z d s \\
= & \int_{0}^{1} \int_{\Omega} X\left(Y^{2}(v+s(u-v))\right) X(u-v)-X\left(\frac{X Y+Y X}{2}\right)(v+s(u-v)) Y(u-v) \\
& -Y\left(\frac{X Y+Y X}{2}\right)(v+s(u-v)) X(u-v)+Y\left(X^{2}(v+s(u-v))\right) Y(u-v) d z d s \\
=\frac{1}{2} & \int_{0}^{1} \int_{\Omega} X\left(Y^{2}(v+s(u-v))\right) X(u-v)-X(X Y+Y X)(v+s(u-v)) Y(u-v) \\
& -Y(X Y+Y X)(v+s(u-v)) X(u-v)+Y\left(X^{2}(v+s(u-v))\right) Y(u-v) d z d s \\
& +\frac{1}{2} \int_{0}^{1} \int_{\Omega} X\left(Y^{2}(v+s(u-v))\right) X(u-v)+Y\left(X^{2}(v+s(u-v))\right) Y(u-v) d z d s \\
= & \int_{0}^{1} \int_{\Omega}(X Y-Y X) Y(v+s(u-v)) X(u-v)-(X Y-Y X) X(v+s(u-v)) Y(u-v) d z d s \\
& \quad-\frac{1}{2} \int_{0}^{1} \int_{\Omega}\left(X^{2} Y(v+s(u-v)) Y(u-v)+Y^{2} X(v+s(u-v)) X(u-v)\right) d z d s \\
& +\frac{1}{2} \int_{0}^{1} \int_{\Omega} X\left(Y^{2}(v+s(u-v))\right) X(u-v)+Y\left(X^{2}(v+s(u-v))\right) Y(u-v) d z d s \\
= & \int_{\Omega}^{1}-2 \partial_{t} Y(v+s(u-v)) X(u-v)+2 \partial_{t} X(v+s(u-v)) Y(u-v) d z d s \\
& +\frac{1}{2} \int_{0}^{1}\left(\left(X Y^{2}-Y^{2} X\right)(v+s(u-v)) X(u-v)+\left(Y X^{2}-X^{2} Y\right)(v+s(u-v)) Y(u-v)\right) d z d s
\end{aligned}
$$

On the other hand,

$$
X Y^{2}-Y^{2} X=X Y^{2}-Y X Y+Y X Y-Y^{2} X=(X Y-Y X) Y+Y(X Y-Y X)=-4 \partial_{t} Y-4 Y \partial_{t}=-8 Y \partial_{t}
$$

and 
Therefore

$$
\begin{aligned}
B & =\int_{0}^{1} \int_{\Omega}-2 \partial_{t} Y(v+s(u-v)) X(u-v)+2 \partial_{t} X(v+s(u-v)) Y(u-v) d z d s \\
& \int_{0}^{1} \int_{\Omega}\left(-4 Y \partial_{t}(v+s(u-v)) X(u-v)+4 X \partial_{t}(v+s(u-v)) Y(u-v)\right) d z d s \\
& =\int_{0}^{1} \int_{\Omega}\left(-6 Y \partial_{t}(v+s(u-v)) X(u-v)+6 X \partial_{t}(v+s(u-v)) Y(u-v)\right) d z d s \\
& =6 \int_{0}^{1} \int_{\Omega} \partial_{t}(v+s(u-v)) Y X(u-v) d z d s-6 \int_{0}^{1} \int_{\partial \Omega} \partial_{t}(v+s(u-v)) X(u-v) v_{Y} d \sigma(z) d s \\
& -6 \int_{0}^{1} \int_{\Omega} \partial_{t}(v+s(u-v)) X Y(u-v) d z d s+6 \int_{0}^{1} \int_{\partial \Omega} \partial_{t}(v+s(u-v)) Y(u-v) v_{X} d \sigma(z) d s \\
& =6 \int_{0}^{1} \int_{\Omega} \partial_{t}(v+s(u-v)) Y X(u-v) d z d s-6 \int_{0}^{1} \int_{\Omega} \partial_{t}(v+s(u-v)) X Y(u-v) d z d s \\
& =6 \int_{0}^{1} \int_{\Omega} \partial_{t}(v+s(u-v))(Y X-X Y)(u-v) d z d s \\
& =24 \int_{0}^{1} \int_{\Omega} \partial_{t}(v+s(u-v)) \partial_{t}(u-v) d z d s \\
& =12 \int_{\Omega} \partial_{t}(u-v) \partial_{t}(u+v) d z d s \\
& =12 \int_{\Omega}\left(\partial_{t} u\right)^{2}-\left(\partial_{t} v\right)^{2} d z d s .
\end{aligned}
$$

This completes the proof of the theorem.

\section{WEAK MAXIMUM PRINCIPLE}

Let $A=\left(a_{i j}\right)$ be a $2 \times 2$ symmetric matrix such that $A \geq 0$, and trace $A>0, a_{i j} \in C(D)$ where $D \subset \mathbb{R}^{3}$ is an open set; $X_{1}=X, X_{2}=Y$, and $L=\sum_{i, j=1}^{2} a_{i j}(\xi) X_{i} X_{j}$.

Theorem 4.1. Let $\Omega$ be a bounded open set in $\mathbb{R}^{3}$, and $w \in C^{2}(\Omega)$. If $L w \geq 0$ in $\Omega$ and $\lim \sup _{\xi \rightarrow \xi_{0}} w(\xi) \leq 0$ for each $\xi_{0} \in \partial \Omega$, then $w \leq 0$ in $\Omega$.

To prove this theorem we need two lemmas.

Lemma 4.2. Let $\Omega \subset \mathbb{R}^{3}$ be an open bounded set, and $w \in C(\Omega)$. Then there exists $\xi_{0} \in \bar{\Omega}$ such that $\sup _{\Omega \cap B\left(\xi_{0}, \rho\right)} w=\sup _{\Omega} w$ for every $\rho>0$, where $B\left(\xi_{0}, \rho\right)$ is the Euclidean ball with radius $\rho$ and center $\xi_{0}$.

Lemma 4.3. Let $\Omega$ be open and bounded. There exists a function $w_{0} \in C^{2}(\Omega)$ such that $w_{0}>0$ and $L w_{0}<0$ in $\Omega$.

Proof. Let $\lambda>0$ and choose $M \in \mathbb{R}$ such that $\sup _{\xi \in \Omega} e^{\lambda x+\lambda y}<M ; \xi=(x, y, t)$. Let $w_{0}=M-e^{\lambda x+\lambda y}$. Then $w_{0}>0$ in $\Omega, X_{1} w_{0}=-\lambda e^{\lambda x}, X_{1}^{2} w_{0}=-\lambda^{2} e^{\lambda x}, X_{2} w_{0}=-\lambda e^{\lambda y}$, 
$X_{2}^{2} w_{0}=-\lambda^{2} e^{\lambda y}$, and $X_{1} X_{2} w_{0}=X_{2} X_{1} w_{0}=0$. Hence $L w_{0}=-\lambda^{2}\left(a_{11} e^{\lambda x}+a_{22} e^{\lambda y}\right)<0$ in $\Omega$.

Proof of Theorem 4.1. First assume that $L w>0$ in $\Omega$. By Lemma 4.2, there exists $\xi_{0} \in \bar{\Omega}$ such that $\sup _{\Omega \cap B\left(\xi_{0}, \rho\right)} w=\sup _{\Omega} w$ for every $\rho>0$. If $\xi_{0} \in \Omega$, then $w\left(\xi_{0}\right)=\sup _{\Omega} w$ and so $D w\left(\xi_{0}\right)=0$ and $D^{2} w\left(\xi_{0}\right) \leq 0$. Hence

$$
\begin{aligned}
0<L w\left(\xi_{0}\right) & =\operatorname{trace}\left(A\left[\begin{array}{cc}
X^{2} w & X Y w \\
Y X w & Y^{2} w
\end{array}\right]\right)\left(\xi_{0}\right) \\
& =\operatorname{trace}\left(A\left[\begin{array}{cc}
X^{2} w & (X Y w+Y X w) / 2 \\
(X Y w+Y X w) / 2 & Y^{2} w
\end{array}\right]\right)\left(\xi_{0}\right) \\
& =\operatorname{trace}\left(A\left[\begin{array}{ccc}
1 & 0 & 2 y \\
0 & 1 & -2 x
\end{array}\right] D^{2} w\left[\begin{array}{cc}
1 & 0 \\
0 & 1 \\
2 y & -2 x
\end{array}\right]\right)\left(\xi_{0}\right) \\
& =\operatorname{trace}\left(\left[\begin{array}{cc}
1 & 0 \\
0 & 1 \\
2 y & -2 x
\end{array}\right] A\left[\begin{array}{ccc}
1 & 0 & 2 y \\
0 & 1 & -2 x
\end{array}\right] D^{2} w\right)\left(\xi_{0}\right) \\
& =\operatorname{trace}\left(\tilde{A} D^{2} w\right)\left(\xi_{0}\right) \leq 0,
\end{aligned}
$$

since $\tilde{A} \geq 0$ and $D^{2} w\left(\xi_{0}\right) \leq 0$. This is a contradiction. Hence $\xi_{0} \in \partial \Omega$ and consequently $w \leq 0$ in $\Omega$. If $L w \geq 0$ in $\Omega$, then for each $\varepsilon>0$ we set $w_{\varepsilon}=w-\varepsilon w_{0}$ with $w_{0}$ as in Lemma 4.3. We have $L w_{\varepsilon}=L w-\varepsilon L w_{0}>0$ and $\lim \sup _{\xi \rightarrow \xi_{0}} w_{\varepsilon}(\xi) \leq \lim \sup _{\xi \rightarrow \xi_{0}} w(\xi) \leq 0$ for each $\xi_{0} \in \partial \Omega$. By the previous argument, $w_{\varepsilon} \leq 0$ in $\Omega$ for each $\varepsilon>0$, and so $w \leq 0$.

Let

$$
\mathcal{H}^{*}(u)=\left[\begin{array}{cc}
Y^{2} u & -(X Y u+Y X u) / 2 \\
-(X Y u+Y X u) / 2 & X^{2} u
\end{array}\right] .
$$

We have

$$
\operatorname{det} \mathcal{H}(u)=\frac{1}{2} \operatorname{trace}\left(\mathcal{H}^{*}(u) \mathcal{H}(u)\right)
$$

and

$$
\operatorname{trace}\left(\mathcal{H}^{*}(u) \mathcal{H}(v)\right)=\operatorname{trace}\left(\mathcal{H}^{*}(v) \mathcal{H}(u)\right)
$$

From Theorem 4.1 we obtain the following comparison principle.

Proposition 4.4. Let $\Omega \subset \mathbb{R}^{3}$ be an open bounded set, $u, v \in C^{2}(\Omega)$ such that $u+v$ is $\mathcal{H}$-convex, and trace $\{\mathcal{H}(u+v)\}>0$. If $\operatorname{det} \mathcal{H}(u) \geq \operatorname{det} \mathcal{H}(v)$ in $\Omega$ and $u \leq v$ on $\partial \Omega$, then $u \leq v$ in $\Omega$. 
Proof. We have

$$
\begin{aligned}
0 & \leq \operatorname{det} \mathcal{H}(u)-\operatorname{det} \mathcal{H}(v) \\
& =\frac{1}{2}\left(\operatorname{trace}\left(\mathcal{H}^{*}(u) \mathcal{H}(u)\right)-\operatorname{trace}\left(\mathcal{H}^{*}(v) \mathcal{H}(v)\right)\right) \\
& =\frac{1}{2}\left(\operatorname{trace}\left(\mathcal{H}^{*}(u) \mathcal{H}(u-v)\right)+\operatorname{trace}\left(\left(\mathcal{H}^{*}(u)-\mathcal{H}^{*}(v)\right) \mathcal{H}(v)\right)\right) \\
& =\frac{1}{2}\left(\operatorname{trace}\left(\mathcal{H}^{*}(u) \mathcal{H}(u-v)\right)+\operatorname{trace}\left(\mathcal{H}^{*}(v) \mathcal{H}(u-v)\right)\right) \\
& =\frac{1}{2} \operatorname{trace}\left(\mathcal{H}^{*}(u+v) \mathcal{H}(u-v)\right) \\
& =\frac{1}{2} \operatorname{trace}\left(\mathcal{H}^{*}(u+v) \mathcal{H}(w)\right),
\end{aligned}
$$

where $w=u-v \leq 0$ on $\partial \Omega$. Applying Theorem 4.1 to $w$ with $A=\mathcal{H}^{*}(u+v)$, the proposition follows.

4.1. A comparison Principle. As a consequence of Proposition 4.4 we get that "cones" that agreeing with an $\mathcal{H}$-convex function $u$ on the boundary of a ball $B$ are above $u$ inside $B$.

Proposition 4.5. Let $d\left(\xi, \xi_{0}\right)=\left\|\xi_{0}^{-1} \circ \xi\right\|,\|\xi\|=\left(\left(x^{2}+y^{2}\right)^{2}+t^{2}\right)^{1 / 4}, \xi=(x, y, t), \Omega=\{\xi \in$ $\left.\mathbb{R}^{3}: 0<d\left(\xi, \xi_{0}\right)<R\right\}$, and $v(\xi)=m\left(\frac{d\left(\xi, \xi_{0}\right)}{R}-1\right)$. If $m \geq 0$, then $v$ is $\mathcal{H}$-convex in $\Omega$, $\operatorname{det} \mathcal{H}(v)=0$ in $\Omega$, and $\operatorname{det} \mathcal{H}(v)$ is integrable in $\bar{\Omega}$.

Proof. If $\zeta \in \mathbb{R}^{3}$ and $g(\xi)=f(\zeta \circ \xi)$, then $X g(\xi)=(X f)(\zeta \circ \xi)$ and $Y g(\xi)=(Y f)(\zeta \circ \xi)$. Therefore we can assume that $\xi_{0}=0$. Let $r=\left(x^{2}+y^{2}\right)^{2}+t^{2}$ and $h \in C^{1}((0,+\infty))$. Then $X r=4 x^{3}+4 x y^{2}+4 y t, Y r=4 y x^{2}+4 y^{3}-4 x t, X^{2} r=Y^{2} r=12\left(x^{2}+y^{2}\right), Y X r=$ $4 t$, and $X Y r=-4 t$. If $u(x, y, t)=h(r)$, then $X u=h^{\prime}(r) X r, Y u=h^{\prime}(r) Y r, X^{2} u=$ $h^{\prime \prime}(r)(X r)^{2}+h^{\prime}(r) X^{2} r, Y^{2} u=h^{\prime \prime}(r)(Y r)^{2}+h^{\prime}(r) Y^{2} r, X Y u=h^{\prime \prime}(r) X r Y r+h^{\prime}(r) X Y r$, $Y X u=h^{\prime \prime}(r) Y r X r+h^{\prime}(r) Y X r$. Thus

$$
\operatorname{det} \mathcal{H}(u)=48\left(x^{2}+y^{2}\right)^{2}\left\{4 r h^{\prime \prime}(r)+3 h^{\prime}(r)\right\} h^{\prime}(r) .
$$

Therefore $\operatorname{det} \mathcal{H}(u)=0$ if $h^{\prime}(r)=0$ or $4 r h^{\prime \prime}(r)+3 h^{\prime}(r)=0$, that is, $h(r)=C$ or $h(r)=r^{1 / 4}$. If $h(r)=r^{1 / 4}$, then $X^{2} h(r)=3 r^{-7 / 4}\left(y\left(x^{2}+y^{2}\right)-x t\right)^{2} \geq 0$ and $Y^{2} h(r)=$ $3 r^{-7 / 4}\left(x\left(x^{2}+y^{2}\right)+y t\right)^{2} \geq 0$, and o $r^{1 / 4}$ is $\mathcal{H}$-convex in $\mathbb{R}^{3} \backslash\{0\}$.

On the other hand, $\operatorname{det} \mathcal{H}(u) \leq C r^{-1 / 2}$ and so $\int_{r^{1 / 4} \leq R} \operatorname{det} \mathcal{H}(u) d z \leq C \int_{r^{1 / 4} \leq R} r^{-1 / 2} d z=$ $C \int_{0}^{R} \rho^{Q-1} \rho^{-2} d \rho=C R^{2}$, since $Q=4$.

Proposition 4.6. Let $u \in C^{2}(\Omega)$ be $\mathcal{H}$-convex, with $\Omega=\left\{\xi \in \mathbb{R}^{3}: 0<d\left(\xi, \xi_{0}\right)<R\right\}$, and $u \leq 0$ on $\left\{\xi \in \mathbb{R}^{3}: d\left(\xi, \xi_{0}\right)=R\right\}$. Then $u \leq v$, where $v$ is defined in Proposition 4.5 with $m=-u\left(\xi_{0}\right)$.

Proof. Let $\varepsilon>0, \xi_{0}=\left(x_{0}, y_{0}, t_{0}\right), \xi=(x, y, t)$,

$$
u_{\varepsilon}(\xi)=u(\xi)+\varepsilon\left(x^{2}+y^{2}\right)
$$


and

$$
v_{\varepsilon}(\xi)=-(1-\sqrt{\varepsilon}) u\left(\xi_{0}\right)\left(\frac{d\left(\xi, \xi_{0}\right)}{(1-\sqrt{\varepsilon}) R}-1\right) .
$$

We first claim that $u_{\varepsilon}(\xi) \leq v_{\varepsilon}(\xi)$ for all $\xi \in \partial \Omega$ and for all $\varepsilon$ sufficiently small. Indeed, if $\xi=\xi_{0}$, then $u_{\varepsilon}\left(\xi_{0}\right) \leq v_{\varepsilon}\left(\xi_{0}\right)$ if and only if $\sqrt{\varepsilon}\left(x_{0}^{2}+y_{0}^{2}\right) \leq-u\left(\xi_{0}\right)$ which holds for all $\varepsilon$ sufficiently small. On the other hand, if $d\left(\xi, \xi_{0}\right)=R$, then $v_{\varepsilon}(\xi)=-\sqrt{\varepsilon} u\left(\xi_{0}\right)$ and $u_{\varepsilon}(\xi) \leq \varepsilon\left(x^{2}+y^{2}\right) \leq \varepsilon \max _{d\left(\xi, \xi_{0}\right)=R}\left(x^{2}+y^{2}\right)=\varepsilon M$. Hence $u_{\varepsilon}(\xi) \leq v_{\varepsilon}(\xi)$ on $d\left(\xi, \xi_{0}\right)=R$ if $\sqrt{\varepsilon} M \leq-u\left(\xi_{0}\right)$ which again holds for all $\varepsilon$ sufficiently small.

We also have

$$
\operatorname{det} \mathcal{H}\left(u_{\varepsilon}\right)=\operatorname{det} \mathcal{H}(u)+2 \varepsilon \operatorname{trace} \mathcal{H}(u)+4 \varepsilon^{2}>0=\operatorname{det} \mathcal{H}\left(v_{\varepsilon}\right)
$$

in $\Omega$, and trace $\left\{\mathcal{H}\left(u_{\varepsilon}+v_{\varepsilon}\right)\right\}=$ trace $\mathcal{H}(u)+8 \varepsilon+\operatorname{trace} \mathcal{H}\left(v_{\varepsilon}\right)>0$. Therefore from Proposition 4.4 we get $u_{\varepsilon} \leq v_{\varepsilon}$ in $\Omega$, and the proposition follows letting $\varepsilon \rightarrow 0$.

As a consequence of these propositions we get the following extension of Theorem 3.1 needed in the proof of the maximum principle Theorem 5.5.

Theorem 4.7. Let $\Omega=\left\{\xi \in \mathbb{R}^{3}: 0<d\left(\xi, \xi_{0}\right)<R\right\}$, and let $v \in C^{2}\left(\bar{B}_{R}\left(\xi_{0}\right)\right)$ be $\mathcal{H}$-convex in $\Omega$ satisfying $v=0$ on $\partial B_{R}\left(\xi_{0}\right)$ and $\operatorname{set} u(\xi)=-v\left(\xi_{0}\right)\left(\frac{d\left(\xi, \xi_{0}\right)}{R}-1\right)$. Then

$$
\int_{B_{R}\left(\xi_{0}\right)}\left\{\operatorname{det} \mathcal{H}(u)+12\left(\partial_{t} u\right)^{2}\right\} d \xi \leq \int_{B_{R}\left(\xi_{0}\right)}\left\{\operatorname{det} \mathcal{H}(v)+12\left(\partial_{t} v\right)^{2}\right\} d \xi
$$

Proof. From Proposition 4.6 we have that $v \leq u$ in $B_{R}\left(\xi_{0}\right)$. Let $\varepsilon>0$, we claim that

$$
\begin{aligned}
& \int_{B_{R}\left(\xi_{0}\right) \backslash B_{\varepsilon}\left(\xi_{0}\right)}\left\{\operatorname{det} \mathcal{H}(u)+12\left(\partial_{t} u\right)^{2}\right\} d \xi \\
& \leq \int_{B_{R}\left(\xi_{0}\right) \backslash B_{\varepsilon}\left(\xi_{0}\right)}\left\{\operatorname{det} \mathcal{H}(v)+12\left(\partial_{t} v\right)^{2}\right\} d \xi+O\left(\varepsilon^{1 / 4}\right),
\end{aligned}
$$

as $\varepsilon \rightarrow 0$. We may assume by the invariance of the vector fields that $\xi_{0}=0$. Since the functions $u, v$ are both convex and $C^{2}$ except at 0 , we proceed as in the proof of Theorem 3.1 applied to the open set $\Omega_{\varepsilon}=B_{R}(0) \backslash B_{\varepsilon}(0)$. The sum of the integrals $I, I I, I I I$ and $I V$ contains now the boundary terms

$$
\begin{aligned}
& -\int_{d(\xi)=\varepsilon} Y^{2}(u+v) X(u-v) \frac{X d}{|D d|} d \sigma(\xi)-\int_{d(\xi)=\varepsilon} X^{2}(u+v) Y(u-v) \frac{Y d}{|D d|} d \sigma(\xi) \\
& -\int_{d(\xi)=\varepsilon} X Y(u+v) X(u-v) \frac{Y d}{|D d|} d \sigma(\xi)-\int_{d(\xi)=\varepsilon} Y X(u+v) Y(u-v) \frac{X d}{|D d|} d \sigma(\xi),
\end{aligned}
$$

where $d(\xi)=d(\xi, 0)$. We shall prove that each summand is $O\left(\varepsilon^{1 / 4}\right)$. Each of these summands basically have the same behaviour as $\varepsilon \rightarrow 0$. Using the computations used in the 
proof of Proposition 4.5, we see for example that

$$
\begin{aligned}
J= & \int_{d(\xi)=\varepsilon} Y^{2}(u+v) X(u-v) \frac{X d}{|D d|} d \sigma(\xi) \\
& \leq C \int_{d(\xi)=\varepsilon} r^{-11 / 4}\left(x\left(x^{2}+y^{2}\right)+y t\right)^{2}\left(4 x^{3}+4 x y^{2}+4 y t\right)^{2} \frac{d \sigma(\xi)}{|D d|} \\
& \leq C \varepsilon^{-11 / 4} \int_{d(\xi)=\varepsilon} \frac{d \sigma(\xi)}{|D d|} .
\end{aligned}
$$

On the other hand, from the coarea formula

$$
\int_{0}^{t} \int_{d(\xi)=s} \frac{d \sigma(\xi)}{|D d|} d s=\int_{d(\xi) \leq t} d \xi=C t^{4} .
$$

So $\int_{d(\xi)=s} \frac{d \sigma(\xi)}{|D d|}=C s^{3}$ and inserting this value in (4.5) we obtain that $J=O\left(\varepsilon^{1 / 4}\right)$.

Following the method of proof of Theorem 3.1 we integrate by parts once again and we now obtain the boundary terms

$$
\int_{d(\xi)=\varepsilon} X(u+v) \partial_{t}(u-v) \frac{Y d}{|D d|} d \sigma(\xi),
$$

and

$$
\int_{d(\xi)=\varepsilon} Y(u+v) \partial_{t}(u-v) \frac{X d}{|D d|} d \sigma(\xi) .
$$

These integrals can be handled as before obtaining again that they are $O\left(\varepsilon^{1 / 4}\right)$. Therefore (4.4) holds and the theorem follows letting $\varepsilon \rightarrow 0$.

As a consequence of Proposition 4.4 we obtain that $\mathcal{H}$-convex functions are Lipschitz with respect to the distance $d$.

Proposition 4.8. Let $\Omega \subset \mathbb{R}^{3}$ be an open set and $u \in C(\Omega) \mathcal{H}$-convex in $\Omega$. Then for each ball $\bar{B} \subset \Omega$ there exists a constant $C_{B}$ such that $|u(x)-u(y)| \leq C_{B} d(x, y)$ for all $x, y \in B$.

Proof. We can assume that $u \in C^{2}(\Omega)$ and let $B_{d}\left(x_{0}, 2 R\right) \subset \Omega$. Let $y \in B_{d}\left(x_{0}, R\right)$ and $\phi(x)=u(x)-u(y)+\varepsilon\left(\left(x_{1}-y_{1}\right)^{2}+\left(x_{2}-y_{2}\right)^{2}\right) ; x=\left(x_{1}, x_{2}, x_{3}\right), y=\left(y_{1}, y_{2}, y_{3}\right)$, with $x \in B_{d}(y, R)$. We have $\mathcal{H}\left(\phi+C_{\varepsilon} d(\cdot, y)\right)>0$ in $B_{d}(y, R)$ and $\phi(x) \leq C_{\varepsilon} d(x, y)$ for $d(x, y)=R$ where $C_{\varepsilon}=\frac{\operatorname{osc}_{B_{d}\left(x_{0}, 2 R\right)} u+\varepsilon \operatorname{diam}\left(B_{d}\left(x_{0}, 2 R\right)\right)^{2}}{R}$. We have $\operatorname{det} \mathcal{H}(\phi) \geq \operatorname{det} \mathcal{H}(d(\cdot, y))$ in $B_{d}(y, R) \backslash\{y\}$ so by the comparison principle Proposition 4.4 we get that $\phi(x) \leq C_{\varepsilon} d(x, y)$ for $x \in B_{d}(y, R)$. Letting $\varepsilon \rightarrow 0$ we get $u(x)-u(y) \leq C d(x, y)$ for $x \in B_{d}(y, R)$ with $C=\frac{\operatorname{Osc}_{B_{d}\left(x_{0}, 2 R\right)} u}{R}$ and $y \in B_{d}\left(x_{0}, R\right)$. If $x, y \in B_{d}\left(x_{0}, R / 4\right)$, then $x \in B_{d}(y, R / 2)$ and so $y \in B_{d}(x, R)$ and by the previous inequality we get $u(y)-u(x) \leq C d(y, x)=C d(x, y)$. Therefore we obtain $|u(x)-u(y)| \leq C d(x, y)$ for all $x, y \in B_{d}\left(x_{0}, R / 4\right)$. 


\section{Maximum Principle}

Proposition 5.1. Let $u$ be $\mathcal{H}$-convex in $\Omega$ open and bounded. Suppose $u \leq 0$ on $\partial \Omega$. Then $u \leq 0$ in $\Omega$.

Proof. Let $\varepsilon>0$ and $u_{\varepsilon}(x, y, t)=u(x, y, t)+\varepsilon\left(x^{2}+y^{2}\right)$. We have $\mathcal{H}\left(u_{\varepsilon}\right)=\mathcal{H}(u)+2 \varepsilon \mathrm{Id}$, so

$$
\operatorname{det} \mathcal{H}\left(u_{\varepsilon}\right)=\operatorname{det} \mathcal{H}(u)+2 \varepsilon \operatorname{trace} \mathcal{H}(u)+4 \varepsilon^{2} .
$$

Since $\operatorname{det} \mathcal{H}\left(\varepsilon\left(x^{2}+y^{2}\right)\right)=4 \varepsilon^{2}$, we get $\operatorname{det} \mathcal{H}\left(u_{\varepsilon}\right) \geq \operatorname{det} \mathcal{H}\left(\varepsilon\left(x^{2}+y^{2}\right)\right)$ in $\Omega$. Also $u_{\varepsilon} \leq$ $\varepsilon\left(x^{2}+y^{2}\right)$ on $\partial \Omega$, and trace $\left\{\mathcal{H}\left(u_{\varepsilon}+\varepsilon\left(x^{2}+y^{2}\right)\right\}=\operatorname{trace} \mathcal{H}(u)+8 \varepsilon>0\right.$. The proposition then follows from Proposition 4.4.

Proposition 5.2. Let $u$ be $\mathcal{H}$-convex in $\Omega$ open and bounded. Suppose $u \leq 0$ on $\partial \Omega$. Then $u \leq 0$ in $\Omega$. Moreover, if there is $\xi_{0} \in \Omega$ such that $u\left(\xi_{0}\right)=0$ then $u \equiv 0$ in $\Omega$.

Proof. Define

$$
L:=X^{2}+Y^{2}
$$

the Kohn Laplacian on the Heisenberg group. Since $u$ is $\mathcal{H}$-convex in $\Omega$ then trace $\mathcal{H} u=$ $L u \geq 0$. Hence, by the maximum principle for $L$, we get $u \leq 0$ in $\Omega$. Moreover, if there is $\xi_{0} \in \Omega$ such that $u\left(\xi_{0}\right)=0$ then $u$ has a maximum at an interior point and by strong maximum principle for $L$, see [1], we get $u \equiv 0$ in $\Omega$.

The following lemma will be used repeatedly in the proof of Proposition 5.4.

Lemma 5.3. Let $\xi_{0} \in B_{R}(0)$ and $\xi \in \Pi_{\xi_{0}} \cap B_{R}(0)$. Let $\lambda>0$ be such that

$$
\xi^{\prime}=\xi_{0} \circ \delta_{\lambda}\left(\xi_{0}^{-1} \circ \xi\right) \in \Pi_{\xi_{0}} \cap \partial B_{R}(0) .
$$

Suppose $u$ is $\mathcal{H}$-convex in $B_{R}(0)$ and $u=0$ on $\partial B_{R}(0)$. Then:

(1) If $\xi_{0}=\left(x_{0}, y_{0}, t_{0}\right)$ and $\xi=\left(0,0, t_{0}\right)$, then $\lambda \geq 2$ and

$$
u(\xi) \leq \frac{1}{2} u\left(\xi_{0}\right) .
$$

(2) If $0<\alpha, \beta<1, \alpha+\beta<1, \rho\left(\xi_{0}\right) \leq \alpha R$ and $d\left(\xi_{0}, \xi\right) \leq \beta R$, then $\lambda \geq \frac{1-\alpha}{\beta}$ and

$$
u(\xi) \leq \frac{1-\alpha-\beta}{1-\alpha} u\left(\xi_{0}\right) .
$$

Proof. To prove the first part of (1), if $\eta=(x, y, t) \in \Pi_{\xi_{0}}$, then we have that

$$
\xi_{0} \circ \delta_{\lambda}\left(\xi_{0}^{-1} \circ \eta\right)=\left(x_{0}+\lambda\left(x-x_{0}\right), y_{0}+\lambda\left(y-y_{0}\right), t_{0}+\lambda\left(t-t_{0}\right)\right),
$$

in particular, $\xi^{\prime}=\left((1-\lambda) x_{0},(1-\lambda) y_{0}, t_{0}\right)$. Hence

$$
\begin{aligned}
R^{4} & =\rho\left(\xi^{\prime}\right)^{4}=\left((1-\lambda)^{2} x_{0}^{2}+(1-\lambda)^{2} y_{0}^{2}\right)^{2}+t_{0}^{2} \\
& =(1-\lambda)^{4}\left(x_{0}^{2}+y_{0}^{2}\right)^{2}+\rho\left(\xi_{0}\right)^{4}-\left(x_{0}^{2}+y_{0}^{2}\right)^{2} \\
& \leq\left((1-\lambda)^{4}-1\right)\left(x_{0}^{2}+y_{0}^{2}\right)^{2}+R^{4},
\end{aligned}
$$

and so $|1-\lambda| \geq 1$. Since $\lambda>0$, it follows that $\lambda \geq 2$. 
To prove the first part of (2) we write

$$
\begin{aligned}
R & =\rho\left(\xi^{\prime}\right)=\rho\left(\left(\xi_{0}^{-1}\right)^{-1} \circ \delta_{\lambda}\left(\xi_{0}^{-1} \circ \xi\right)\right) \leq \rho\left(\xi_{0}^{-1}\right)+\rho\left(\delta_{\lambda}\left(\xi_{0}^{-1} \circ \xi\right)\right) \\
& =\rho\left(\xi_{0}\right)+\lambda \rho\left(\xi_{0}^{-1} \circ \xi\right)=\rho\left(\xi_{0}\right)+\lambda d\left(\xi_{0}, \xi\right) \\
& \leq \alpha R+\lambda \beta R
\end{aligned}
$$

and so $\lambda \geq \frac{1-\alpha}{\beta}$.

To prove (5.6) and (5.7), by definition of $\xi^{\prime}$ we have that $\xi=\xi_{0} \circ \delta_{1 / \lambda}\left(\xi_{0}^{-1} \circ \xi^{\prime}\right)$. From (2.4) and since $u\left(\xi^{\prime}\right)=0$, it follows that $u(\xi) \leq\left(1-\frac{1}{\lambda}\right) u\left(\xi_{0}\right)$. Thus (5.6) and (5.7) follow since $u \leq 0$ in $B_{R}(0)$.

Proposition 5.4. Let $u$ be $\mathcal{H}$-convex and $u=0$ on $\partial B_{R}(0)$. Given $\xi_{0} \in B_{R}(0)$ there exists a positive constant $c<1$, depending on $d\left(\xi_{0}, \partial B_{R}(0)\right)$, such that

$$
u(0) \leq c u\left(\xi_{0}\right) \text {. }
$$

Proof. Let $\xi_{0}=\left(x_{0}, y_{0}, t_{0}\right)$ and $\xi_{1}=\exp \left(-x_{0} X-y_{0} Y\right)\left(\xi_{0}\right)=\left(0,0, t_{0}\right) \in \Pi_{\xi_{0}}$. We obviously have that $d\left(\xi_{1}, \xi_{0}\right)=\sqrt{x_{0}^{2}+y_{0}^{2}} \leq d\left(0, \xi_{0}\right)<R$. Applying Lemma 5.3(1) with $\xi_{0} \rightsquigarrow \xi_{0}$ and $\xi \rightsquigarrow \xi_{1}$ we get that

$$
u\left(\xi_{1}\right) \leq \frac{1}{2} u\left(\xi_{0}\right)
$$

We shall prove that there exists a constant $C_{1}>0$ depending only of the distance from $\xi_{1}$ to $\partial B_{R}(0)$ such that

$$
u(0) \leq C_{1} u\left(\xi_{1}\right) .
$$

To prove (5.9) we may assume $\xi_{1} \neq 0$, and consider two cases.

Case 1. $d\left(\xi_{1}, 0\right)=\left|t_{0}\right|^{1 / 2} \leq R / 2$.

$$
\text { If } \begin{aligned}
t_{0}> & 0, \text { define } \sigma=\frac{\sqrt{t_{0}}}{2} \text { and put } \\
& \xi_{2}=\exp (\sigma X) \xi_{1}=\left(\sigma, 0, t_{0}\right), \\
\xi_{3} & =\exp (\sigma Y) \xi_{2}=\left(\sigma, \sigma, t_{0}-2 \sigma^{2}\right), \\
& \xi_{4}=\exp (-\sigma X) \xi_{3}=\left(0, \sigma, t_{0}-2 \sigma^{2}-2 \sigma^{2}\right)=\left(0, \sigma, t_{0}-4 \sigma^{2}\right)=(0, \sigma, 0) .
\end{aligned}
$$

By our choice of $\sigma$ we have

$$
\exp (-\sigma Y) \xi_{4}=\left(0,0, t_{0}-4 \sigma^{2}\right)=0
$$

Let us remark that

We have

$$
\sigma=\frac{1}{2} d\left(\xi_{1}, 0\right) \leq R / 4
$$

$$
\begin{gathered}
d\left(\xi_{1}, \xi_{2}\right)=d\left(\xi_{2}, \xi_{3}\right)=d\left(\xi_{3}, \xi_{4}\right)=\sigma ; \\
\rho\left(\xi_{2}\right)=17^{1 / 4} \sigma ; \quad \rho\left(\xi_{3}\right)=8^{1 / 4} \sigma ; \quad \rho\left(\xi_{4}\right)=\sigma .
\end{gathered}
$$


Hence $\xi_{2}, \xi_{3}, \xi_{4} \in B_{R}$. Applying Lemma 5.3(2) with $\xi_{0} \rightsquigarrow \xi_{1}, \xi \rightsquigarrow \xi_{2}, \alpha=1 / 2$, and $\beta=1 / 4$ we get that

$$
u\left(\xi_{2}\right) \leq \frac{1}{2} u\left(\xi_{1}\right)
$$

Next, applying Lemma 5.3(2) with $\xi_{0} \rightsquigarrow \xi_{2}, \xi \rightsquigarrow \xi_{3}, \alpha=17^{1 / 4} / 4$, and $\beta=1 / 4$, we get that

$$
u\left(\xi_{3}\right) \leq \frac{3-17^{1 / 4}}{4-17^{1 / 4}} u\left(\xi_{2}\right)<\frac{3}{8} u\left(\xi_{2}\right)
$$

Applying once again Lemma 5.3(2) now with $\xi_{0} \rightsquigarrow \xi_{3}$ and $\xi \rightsquigarrow \xi_{4}, \alpha=8^{1 / 4} / 4, \beta=1 / 4$, we get that

$$
u\left(\xi_{4}\right) \leq \frac{3-8^{1 / 4}}{4-8^{1 / 4}} u\left(\xi_{3}\right)<\frac{1}{2} u\left(\xi_{3}\right)
$$

Define

$$
\xi^{(4)}=\xi_{4} \circ \delta_{\lambda}\left(\xi_{4}^{-1}\right) \in \Pi_{\xi_{4}}
$$

and choose $\lambda>0$ such that $\xi^{(4)} \in \partial B_{R}$. Applying Lemma 5.3(2) now with $\xi_{0} \leadsto \xi_{4}$ and $\xi \rightsquigarrow 0, \alpha=1 / 4, \beta=1 / 4$, we get that

$$
u(0) \leq \frac{2}{3} u\left(\xi_{4}\right)
$$

This completes the proof of (5.9) for $t_{0}>0$.

$$
\begin{aligned}
& \text { If } t_{0}<0 \text {, define } \sigma=\frac{\sqrt{-t_{0}}}{2} \text { and put } \\
& \xi_{2}=\exp (\sigma Y) \xi_{1}=\left(0, \sigma, t_{0}\right), \\
& \xi_{3}=\exp (\sigma X) \xi_{2}=\left(\sigma, \sigma, t_{0}+2 \sigma^{2}\right) \text {, } \\
& \xi_{4}=\exp (-\sigma Y) \xi_{3}=\left(\sigma, 0, t_{0}+4 \sigma^{2}\right) \text {. }
\end{aligned}
$$

By our choice of $\sigma$ we have

$$
\exp (-\sigma X) \xi_{4}=\left(0,0, t_{0}+4 \sigma^{2}\right)=0 .
$$

Then, arguing as in case $t_{0}>0$, we get (5.9).

Case 2. $R / 2<d\left(\xi_{1}, 0\right)=\left|t_{0}\right|^{1 / 2}<R$.

Define

$$
d:=\frac{d\left(\xi_{1}, \partial B_{R}\right)}{\sqrt{6}}=\frac{\sqrt{R^{2}-\left|t_{0}\right|}}{\sqrt{6}}
$$

Obviously $d^{2}<R^{2} / 8$. It is not restrictive to assume $t_{0}>0$. We first prove that there exists a universal constant $0<C_{2}<1$ such that

$$
u\left(0,0, t_{0}-4 d^{2}\right) \leq C_{2} u\left(\xi_{1}\right)
$$


Let

$$
\begin{aligned}
& \xi_{1}=\left(0,0, t_{0}\right) \\
& \xi_{2}=\exp (d X)\left(\xi_{1}\right)=\left(d, 0, t_{0}\right) \\
& \xi_{3}=\exp (d Y)\left(\xi_{2}\right)=\left(d, d, t_{0}-2 d^{2}\right) \\
& \xi_{4}=\exp (-d X)\left(\xi_{3}\right)=\left(0, d, t_{0}-4 d^{2}\right) \\
& \xi_{5}=\exp (-d Y)\left(\xi_{4}\right)=\left(0,0, t_{0}-4 d^{2}\right) .
\end{aligned}
$$

We have $\xi_{i+1} \in \Pi_{\xi_{i}}$ for $i=1,2,3,4$. Let

$$
\xi_{2}^{(1)}=\exp (\lambda d X)\left(\xi_{1}\right)=\left(\lambda d, 0, t_{0}\right)=\xi_{1} \circ \delta_{\lambda}\left(\xi_{1}^{-1} \circ \xi_{2}\right),
$$

with $\lambda>0$ such that $\xi_{2}^{1} \in \prod_{\xi_{1}} \cap \partial B_{R}$. Then

$$
R^{4}=\rho\left(\xi_{2}^{(1)}\right)=\lambda^{4} d^{4}+t_{0}^{2}=\lambda^{4} d^{4}+\left(R^{2}-6 d^{2}\right)^{2}=\left(\lambda^{4}+36\right) d^{4}+R^{4}-12 d^{2} R^{2},
$$

and so

$$
12 R^{2}=\left(\lambda^{4}+36\right) d^{2} \leq\left(\lambda^{4}+36\right) R^{2} / 8
$$

which yields $\lambda>2$. Hence,

$$
u\left(\xi_{2}\right) \leq(1 / 2) u\left(\xi_{1}\right)
$$

We have

$$
\begin{aligned}
\rho\left(\xi_{2}\right)^{4} & =d^{4}+t_{0}^{2}=d^{4}+\left(R^{2}-6 d^{2}\right)^{2} \\
& =37 d^{4}+R^{4}-12 R^{2} d^{2}=d^{2}\left(37 d^{2}-12 R^{2}\right)+R^{4} \\
& \leq d^{2}(37 / 8-12) R^{2}+R^{4}=\left(\frac{1}{8}\left(\frac{37}{8}-12\right)+1\right) R^{4}<R^{4},
\end{aligned}
$$

and

$$
d\left(\xi_{2}, \xi_{3}\right)=d \leq \frac{1}{\sqrt{8}} R
$$

If

$$
\xi_{3}^{(2)}=\exp (\lambda d Y)\left(\xi_{2}\right)=\left(d, \lambda d, t_{0}-2 \lambda d^{2}\right)=\xi_{2} \circ \delta_{\lambda}\left(\xi_{2}^{-2} \circ \xi_{3}\right)
$$

and we pick $\lambda>0$ such that $\xi_{3}^{2} \in \Pi_{\xi_{2}} \cap \partial B_{R}$, then applying Lemma 5.3(2) with $\xi_{0} \rightsquigarrow \xi_{2}$, $\xi \rightsquigarrow \xi_{3}, \alpha=\sqrt[4]{\frac{1}{8}\left(\frac{37}{8}-12\right)+1}$, and $\beta=\frac{1}{\sqrt{8}}$, we get that

$$
u\left(\xi_{3}\right) \leq \frac{\sqrt{8}-\sqrt[4]{5}-1}{\sqrt{8}-\sqrt[4]{5}} u\left(\xi_{2}\right)<\frac{1}{5} u\left(\xi_{2}\right) .
$$

Next,

$$
\begin{aligned}
\rho\left(\xi_{3}\right)^{4} & =\left(2 d^{2}\right)^{2}+\left(t_{0}-2 d^{2}\right)^{2}=4 d^{4}+\left(R^{2}-8 d^{2}\right)^{2}=68 d^{4}-16 R^{2} d^{2}+R^{4} \\
& \leq d^{2} R^{2}(68 / 8-16)+R^{4} \leq\left(\frac{1}{8}\left(\frac{68}{8}-16\right)+1\right) R^{4}<R^{4}
\end{aligned}
$$


and

$$
d\left(\xi_{3}, \xi_{4}\right)=d \leq \frac{1}{\sqrt{8}} R
$$

Let

$$
\xi_{4}^{(3)}=\exp (-\lambda d X)\left(\xi_{3}\right)=\left((1-\lambda) d, d, t_{0}-2 d^{2}-2 \lambda d^{2}\right)=\xi_{3} \circ \delta_{\lambda}\left(\xi_{3}^{-1} \circ \xi_{4}\right),
$$

with $\lambda>0$ such that $\xi_{4}^{(3)} \in \partial B_{R} \cap \Pi_{\xi_{3}}$. Applying Lemma 5.3(2) with $\xi_{0} \rightsquigarrow \xi_{3}, \xi \rightsquigarrow \xi_{4}$,

$$
\begin{aligned}
& \alpha=\sqrt[4]{\frac{1}{8}\left(\frac{68}{8}-16\right)+1}=\frac{1}{2} \text {, and } \beta=\frac{1}{\sqrt{8}} \text {, we get that } \\
& u\left(\xi_{4}\right)<\frac{1}{4} u\left(\xi_{3}\right) .
\end{aligned}
$$

We have

$$
\begin{aligned}
\rho\left(\xi_{4}\right)^{4} & =d^{4}+\left(t_{0}-4 d^{2}\right)^{2}=d^{4}+\left(R^{2}-10 d^{2}\right)^{2}=101 d^{4}+R^{4}-20 R^{2} d^{2} \\
& =\left(101 d^{2}-20 R^{2}\right) d^{2}+R^{4} \leq(101 / 8-20) R^{2} d^{2}+R^{4} \\
& \leq\left(\frac{1}{8}\left(\frac{101}{8}-20\right)+1\right) R^{4}<R^{4}
\end{aligned}
$$

and

$$
d\left(\xi_{4}, \xi_{5}\right)=d \leq \frac{1}{\sqrt{8}} R
$$

Letting

$$
\xi_{5}^{(4)}=\exp (-\lambda d Y)\left(\xi_{4}\right)=\left(0,(1-\lambda) d, t_{0}-4 d^{2}\right)=\xi_{4} \circ \delta_{\lambda}\left(\xi_{4}^{-1} \circ \xi_{5}\right)
$$

with $\lambda>1$ such that $\xi_{5}^{(4)} \in \Pi_{\xi_{4}} \cap \partial B_{R}$, and applying Lemma 5.3(2) with $\xi_{0} \rightsquigarrow \xi_{4}, \xi \rightsquigarrow \xi_{5}$, $\alpha=\sqrt[4]{\frac{1}{8}\left(\frac{101}{8}-20\right)+1}=\frac{\sqrt[4]{5}}{\sqrt{8}}$, and $\beta=\frac{1}{\sqrt{8}}$, we get that

$$
u\left(\xi_{5}\right) \leq \frac{1}{5} u\left(\xi_{4}\right)
$$

Thus, inequality (5.11) follows.

We now iterate the inequality (5.11). Let $d_{0}=d$ (defined in 5.10), $t_{1}=t_{0}-4 d_{0}^{2}$, and in general

$$
t_{j+1}=t_{j}-4 d_{j}^{2}, \quad \text { and } \quad d_{j}^{2}=\frac{R^{2}-t_{j}}{6} .
$$

We have $d_{j+1}^{2}=\frac{R^{2}-t_{j+1}}{6}=\frac{R^{2}-t_{j}+4 d_{j}^{2}}{6}=\left(1+\frac{2}{3}\right) d_{j}^{2}$. Thus,

$$
\begin{aligned}
t_{N+1}=t_{0}-4 \sum_{j=0}^{N} d_{j}^{2} & =t_{0}-4 d_{0}^{2} \sum_{j=0}^{N}\left(1+\frac{2}{3}\right)^{j} \\
& =t_{0}-\left(R^{2}-t_{0}\right)\left(\left(1+\frac{2}{3}\right)^{N+1}-1\right) .
\end{aligned}
$$


Pick $N$ such that

$$
t_{N} \leq \frac{R^{2}}{4}<t_{N-1}
$$

which amounts

$$
N-1<\ln \left[\frac{3 R^{2}}{4\left(R^{2}-t_{0}\right)}\right]^{1 / \ln (1+2 / 3)} \leq N
$$

We have $t_{N}<t_{N-1}<\cdots<t_{1}<t_{0}$ and it is easy to check from (5.12), the choice of $N$ and 5.10 that $t_{N} \geq-R^{2} / 4$. Therefore $\left(0,0, t_{j}\right) \in B_{R}(0) \backslash B_{R / 2}(0)$ for $0 \leq j \leq N-1$ and $\left(0,0, t_{N}\right) \in B_{R / 2}(0)$. Iterating (5.11) $N$ times, then yields

$$
u\left(0,0, t_{N}\right) \leq C_{1}^{N} u\left(\xi_{1}\right)
$$

Since $0<C_{1}<1$, there is $\gamma>0$ such that $C_{1}=e^{-\gamma}$, and from (5.13) we obtain

$$
\begin{aligned}
u\left(0,0, t_{N}\right) & \leq C_{1} \exp \left(-\gamma \ln \left[\frac{3 R^{2}}{4\left(R^{2}-t_{0}\right)}\right]^{1 / \ln (1+2 / 3)}\right) u\left(\xi_{1}\right) \\
& =C_{1}\left[\frac{4\left(R^{2}-t_{0}\right)}{3 R^{2}}\right]^{\gamma / \ln (1+2 / 3)} u\left(\xi_{1}\right) .
\end{aligned}
$$

Since $\left(0,0, t_{N}\right) \in B_{R / 2}(0)$, we can apply (5.9) to get $u(0) \leq C_{1} u\left(0,0, t_{N}\right)$. Consequently,

$$
u(0) \leq C_{1}^{2}\left[\frac{4\left(R^{2}-t_{0}\right)}{3 R^{2}}\right]^{\gamma / \ln (1+2 / 3)} u\left(\xi_{1}\right),
$$

which completes the proof of (5.9) in Case 2.

Finally, combining (5.8) and (5.9) we obtain the proposition.

Theorem 5.5. Let $u \in C^{2}\left(B_{R}\right)$ be $\mathcal{H}$-convex, $u=0$ on $\partial B_{R}$. If

$$
u\left(\xi_{0}\right)=\min _{B_{R}} u
$$

then there exists a positive constant $c$, depending on $d\left(\xi_{0}, \partial B_{R}\right)$, such that

$$
\left|u\left(\xi_{0}\right)\right|^{2} \leq c \int_{B_{R}}\left(\operatorname{det} \mathcal{H}(u)+12 u_{t}^{2}\right) d z
$$

Proof. Define

$$
u(0)=-m
$$

and

$$
v(\zeta)=m\left(\frac{d(\zeta, 0)}{R}-1\right)
$$

We have $v=u=0$ on $\partial B_{R}, v$ is $\mathcal{H}$-convex in $B_{R}$ and $v \geq u$ in $B_{R}$. From the comparison principle, Theorem 4.7 , we then get

$$
\int_{B_{R}}\left\{\operatorname{det} \mathcal{H}(v)+12 v_{t}^{2}\right\} d z \leq \int_{B_{R}}\left\{\operatorname{det} \mathcal{H}(u)+12 u_{t}^{2}\right\} d z .
$$


Moreover,

$$
\begin{aligned}
\int_{B_{R}}\left\{\operatorname{det} \mathcal{H}(v)+12 v_{t}^{2}\right\} d z & =\left(\frac{m}{R}\right)^{2} \int_{B_{R}}\left\{\operatorname{det} \mathcal{H}(d(\zeta, 0))+12\left(\partial_{t} d(\zeta, 0)\right)^{2}\right\} d \zeta \\
& =12\left(\frac{m}{R}\right)^{2} R^{2} \int_{B_{1}}\left(\partial_{t} d(\zeta, 0)\right)^{2} d \zeta \\
& =c_{1} m^{2}
\end{aligned}
$$

with

$$
c_{1}=12 \int_{B_{1}}\left(\partial_{t} d(\zeta, 0)\right)^{2} d \zeta>0
$$

Let

$$
u\left(\xi_{0}\right)=\min _{B_{R}} u=-m_{0} .
$$

By Proposition 5.4 there exists a constant $0<c_{2}<1$ such that

$$
m_{0} \leq \frac{1}{c_{2}} m
$$

Hence,

$$
m_{0}^{2} \leq \frac{1}{c_{2}^{2}} m^{2} \leq \frac{c_{1}}{c_{2}^{2}} \int_{B_{R}}\left\{\operatorname{det} \mathcal{H}(u)+12 u_{t}^{2}\right\} d z
$$

\section{6. $\mathcal{H}$-MeAsures}

6.1. Oscillation estimate. In this section we prove that if $u$ is $\mathcal{H}$-convex, we can control the integral of $\operatorname{det} \mathcal{H}(u)+12\left(u_{t}\right)^{2}$ locally in terms of the oscillation of $u$.

Let us start with a lemma on $\mathcal{H}$-convex functions, which is similar to the Euclidean one for convex functions.

Lemma 6.1. If $u_{1}, u_{2} \in C^{2}(\Omega)$ are $\mathcal{H}$-convex, and $f$ is convex in $\mathbb{R}^{2}$ and nondecreasing in each variable, then the composite function $w=f\left(u_{1}, u_{2}\right)$ is $\mathcal{H}$-convex.

Proof. Assume first that $f \in C^{2}\left(\mathbb{R}^{2}\right)$, and set $X_{1}=X, X_{2}=Y$. We have

$$
\begin{gathered}
X_{j} w=\sum_{p=1}^{2} \frac{\partial f}{\partial u_{p}} X_{j} u_{p}, \\
X_{i} X_{j} w=\sum_{p=1}^{2}\left(\frac{\partial f}{\partial u_{p}} X_{i} X_{j} u_{p}+\sum_{q=1}^{2} \frac{\partial^{2} f}{\partial u_{q} \partial u_{p}} X_{i} u_{q} X_{j} u_{p}\right),
\end{gathered}
$$


and for every $h=\left(h_{1}, h_{2}\right) \in \mathbb{R}^{2}$

$$
\begin{aligned}
\langle\mathcal{H}(w) h, h\rangle & =\sum_{i, j=1}^{2} X_{i} X_{j} w h_{i} h_{j} \\
& =\sum_{p=1}^{2} \frac{\partial f}{\partial u_{p}}\left\langle\mathcal{H}\left(u_{p}\right) h, h\right\rangle+\sum_{p, q=1}^{2} \frac{\partial^{2} f}{\partial u_{q} \partial u_{p}}\left(\sum_{i=1}^{2} X_{i} u_{q} h_{i}\right)\left(\sum_{j=1}^{2} X_{j} u_{p} h_{j}\right) \\
& \geq 0,
\end{aligned}
$$

since $\mathcal{H}\left(u_{p}\right)$ is non negative definite and $\frac{\partial f}{\partial u_{p}} \geq 0$ for $p=1,2$, and the matrix

$$
\left(\frac{\partial^{2} f}{\partial u_{q} \partial u_{p}}\right)_{p, q=1,2}
$$

is non negative definite.

If $f$ is only continuous, then given $h>0$ let

$$
f_{h}(x)=h^{-2} \int_{\mathbb{R}^{2}} \varphi\left(\frac{x-y}{h}\right) f(y) d y,
$$

where $\varphi \in C^{\infty}$ is nonnegative vanishing outside the unit ball of $\mathbb{R}^{2}$, and $\int \varphi=1$. Since $f$ is convex, then $f_{h}$ is convex and by the previous calculation $w_{h}=f_{h}\left(u_{1}, u_{2}\right)$ is $\mathcal{H}$-convex. In particular, $w_{h}$ satisfies Proposition 2.3 and since $w_{h} \rightarrow w$ uniformly on compact sets as $h \rightarrow 0$, we get that $w$ is $\mathcal{H}$-convex.

Proposition 6.2. Let $u \in C^{2}(\Omega)$ be $\mathcal{H}$-convex. For any compact domain $\Omega^{\prime} \Subset \Omega$ there exists a positive constant $C$ depending on $\Omega^{\prime}$ and $\Omega$ and independent of $u$, such that

$$
\int_{\Omega^{\prime}}\left\{\operatorname{det} \mathcal{H}(u)+12\left(u_{t}\right)^{2}\right\} d z \leq C\left(\operatorname{osc}_{\Omega} u\right)^{2} .
$$

Proof. Given $\xi_{0} \in \Omega$ let $B_{R}=B_{R}\left(\xi_{0}\right)$ be a $d$-ball of radius $R$ and center at $\xi_{0}$ such that $B_{R} \subset \Omega$. Let $B_{\sigma R}$ be the concentric ball of radius $\sigma R$, with $0<\sigma<1$. Without loss of generality we can assume $\xi_{0}=0$, because the vector fields $X$ and $Y$ are left invariant with respect to the group of translations. Let $M=\max _{B_{R}} u$, then $u-M \leq 0$ in $B_{R}$. Given $\varepsilon>0$ we shall work with the function $u-M-\varepsilon<-\varepsilon$. In other words, by subtracting a constant, we may assume $u<-\varepsilon$ in $B_{R}$, for each given positive constant $\varepsilon$; $\varepsilon$ will tend to zero at the end of the proof.

Define

$$
m_{0}=\inf _{B_{R}} u
$$

and

$$
v(\xi)=\frac{m_{0}}{\left(1-\sigma^{4}\right) R^{4}}\left(R^{4}-\|\xi\|^{4}\right) .
$$

Obviously $v=0$ on $\partial B_{R}$ and $v=m_{0}$ on $\partial B_{\sigma R}$. We claim that $v$ is $\mathcal{H}$-convex in $B_{R}$ and $v \leq m_{0}$ in $B_{\sigma R}$. Setting $r=\|\xi\|^{4}, h(r)=\frac{m_{0}}{\left(1-\sigma^{4}\right) R^{4}}\left(R^{4}-r\right)$, and following the calculations 
in the proof of Proposition 4.5 we get

$$
\operatorname{det} \mathcal{H}(v)=144\left(x^{2}+y^{2}\right)^{2}\left(\frac{m_{0}}{\left(1-\sigma^{4}\right) R^{4}}\right)^{2} \geq 0,
$$

and

$$
X^{2} h=Y^{2} h=-12\left(x^{2}+y^{2}\right) \frac{m_{0}}{\left(1-\sigma^{4}\right) R^{4}} \geq 0,
$$

because $m_{0}$ is negative. Hence $v$ is $\mathcal{H}$-convex in $B_{R}$. Since $v-m_{0}=0$ on $\partial B_{\sigma R}$, it follows from Proposition 5.1 that $v \leq m_{0}$ in $B_{\sigma R}$. In particular, $v \leq u$ in $B_{\sigma R}$.

Let $\rho \in C_{0}^{\infty}\left(\mathbb{R}^{2}\right)$, radial with support in the Euclidean unit ball, $\int_{\mathbb{R}^{2}} \rho(x) d x=1$, and let

$$
f_{h}\left(x_{1}, x_{2}\right)=h^{-2} \int_{\mathbb{R}^{2}} \rho((x-y) / h) \max \left\{y_{1}, y_{2}\right\} d y_{1} d y_{2} .
$$

We have that

(1) If $x_{1}>x_{2}$, then there exists $h_{0}>0$ and a neighborhood $V$ of $\left(x_{1}, x_{2}\right)$ such that $f_{h}\left(y_{1}, y_{2}\right)=y_{1}$ for all $\left(y_{1}, y_{2}\right) \in V$ and for all $h \leq h_{0} .^{\dagger}$

(2) There exists a positive constant $\alpha$ such that $f_{h}(x, x)=x+\alpha h$ for all $h>0$ and for all $x \in \mathbb{R}^{\ddagger}$

\footnotetext{
${ }^{\dagger}$ If $x_{1}>x_{2}$, then there exists a cube $Q$ centered at $\left(x_{1}, x_{2}\right)$ such that if $\left(z_{1}, z_{2}\right) \in Q$ then $z_{1}>z_{2}$. Hence $x_{1}-y_{1}>x_{2}-y_{2}$ for all $\left|\left(y_{1}, y_{2}\right)\right|<h$ with $h$ sufficiently small. Then

$$
\begin{aligned}
f_{h}\left(x_{1}, x_{2}\right) & =h^{-2} \int_{|y|<h} \rho(y / h)\left(x_{1}-y_{1}\right) d y_{1} d y_{2}=x_{1}-h^{-2} \int_{|y|<h} \rho(y / h) y_{1} d y_{1} d y_{2} \\
& =x_{1}-h \int_{0}^{1} t^{2} \rho(t) \int_{S^{1}} y_{1} d \sigma(y) d t=x_{1} .
\end{aligned}
$$
}

${ }^{\ddagger}$ We have

$$
\begin{aligned}
& f_{h}(x, x)=h^{-2} \int_{|y|<h} \rho(y / h) \max \left\{x-y_{1}, x-y_{2}\right\} d y_{1} d y_{2} \\
& =h^{-2} \int_{|y|<h} \rho(y / h)\left(x+\max \left\{-y_{1},-y_{2}\right\}\right) d y_{1} d y_{2} \\
& =x_{1}+h^{-2} \int_{|y|<h} \rho(y / h) \max \left\{-y_{1},-y_{2}\right\} d y_{1} d y_{2} \\
& =x_{1}+h^{-2} \int_{|y|<h} \rho(y / h) \max \left\{y_{1}, y_{2}\right\} d y_{1} d y_{2} \\
& =x_{1}+h \int_{|y|<1} \rho(y) \max \left\{y_{1}, y_{2}\right\} d y_{1} d y_{2} \\
& =x_{1}+h \int_{0}^{1} t^{2} \rho(t) \int_{S^{1}} \max \left\{y_{1}, y_{2}\right\} d \sigma(y) d t \\
& =x_{1}+h \int_{0}^{1} t^{2} \rho(t) \int_{S^{1}} \frac{\left|y_{1}-y_{2}\right|+y_{1}+y_{2}}{2} d \sigma(y) d t \\
& =x_{1}+h \int_{0}^{1} t^{2} \rho(t) \int_{S^{1}} \frac{\left|y_{1}-y_{2}\right|}{2} d \sigma(y) d t=x_{1}+\alpha h \text {. }
\end{aligned}
$$


(3) For all $h>0, f_{h}\left(\cdot, x_{2}\right)$ is nondecreasing for each $x_{2}$ and $f_{h}\left(x_{1}, \cdot\right)$ is nondecreasing for each $x_{1}$.

Define

$$
w_{h}=f_{h}(u, v) .
$$

From Lemma $6.1 w_{h}$ is $\mathcal{H}$-convex in $B_{R}$. If $y \in B_{\sigma R}$ then $v(y) \leq u(y)$. If $v(y)<u(y)$ then $f_{h}(u, v)(y)=u(y)$ for $h$ sufficiently small; and if $v(y)=u(y)$, then $f_{h}(u, v)(y)=u(y)+\alpha h$. Hence

$$
\begin{aligned}
\int_{B_{\sigma R}}\left\{\operatorname{det} \mathcal{H}(u)+12\left(\partial_{t} u\right)^{2}\right\} d z & =\int_{B_{\sigma R}}\left\{\operatorname{det} \mathcal{H}\left(w_{h}\right)+12\left(\left(w_{h}\right)_{t}\right)^{2}\right\} d z \\
& \leq \int_{B_{R}}\left\{\operatorname{det} \mathcal{H}\left(w_{h}\right)+12\left(\left(w_{h}\right)_{t}\right)^{2}\right\} d z
\end{aligned}
$$

Now notice that $f_{h}(u, v) \geq v$ in $B_{R}$ for all $h$ sufficiently small. In addition, $u<0$ and $v=0$ on $\partial B_{R}$ so $f_{h}(u, v)=0$ on $\partial B_{R}$. Then we can apply Theorem 3.1 to $w_{h}$ and $v$ to get

$$
\begin{aligned}
\int_{B_{R}}\left\{\operatorname{det} \mathcal{H}\left(w_{h}\right)+12\left(\partial_{t} w_{h}\right)^{2}\right\} d z & \leq \int_{B_{R}}\left\{\operatorname{det} \mathcal{H}(v)+12\left(v_{t}\right)^{2}\right\} d z \\
& =48\left(\frac{m_{0}}{(1-\sigma) R^{4}}\right)^{2} \int_{B_{R}}\left(3\left(x^{2}+y^{2}\right)^{2}+t^{2}\right) d z \\
& =48\left(\frac{m_{0}}{(1-\sigma)}\right)^{2} \int_{B_{1}}\left(3\left(x^{2}+y^{2}\right)^{2}+t^{2}\right) d z .
\end{aligned}
$$

This inequality combined with (6.16) yields

$$
\int_{B_{\sigma R}}\left\{\operatorname{det} \mathcal{H}(u)+12\left(\partial_{t} u\right)^{2}\right\} d z \leq C\left(m_{0}\right)^{2} \leq C\left(\operatorname{osc}_{B_{R}} u+\varepsilon\right)^{2} .
$$

The inequality (6.14) then follows letting $\varepsilon \rightarrow 0$ and covering $\Omega^{\prime}$ with balls.

Corollary 6.3. Let $u \in C^{2}(\Omega)$ be $\mathcal{H}$-convex. For any compact domain $\Omega^{\prime} \Subset \Omega$ there exists a positive constant $C$, independent of $u$, such that

$$
\int_{\Omega^{\prime}} \operatorname{det} \mathcal{H}(u) d z \leq C\left(\operatorname{osc}_{\Omega} u\right)^{2} .
$$

Corollary 6.4. Let $u \in C^{2}(\Omega)$ be $\mathcal{H}$-convex. For any compact domain $\Omega^{\prime} \Subset \Omega$ there exists a positive constant $C$, independent of $u$, such that

$$
\int_{\Omega^{\prime}} \operatorname{trace} \mathcal{H}_{2}(u) d z \leq C R^{2} \operatorname{osc}_{\Omega} u .
$$

6.2. Measure generated by an $\mathcal{H}$-convex function. We shall prove that the notion $\int \operatorname{det} \mathcal{H}(u)+u_{t}^{2}$ can be extended for continuous and $\mathcal{H}$-convex functions as a Borel measure. We call this measure the $\mathcal{H}$-measure associated with $u$, and we shall show that the map $u \in C(\Omega) \rightarrow \mu(u)$ is weakly continuous on $C(\Omega)$. 
Theorem 6.5. Given an $\mathcal{H}$-convex function $u \in C(\Omega)$ there exists a unique Borel measure $\mu(u)$ such that when $u \in C^{2}(\Omega)$,

$$
\mu(u)(E)=\int_{E}\left\{\operatorname{det} \mathcal{H}(u)+12 u_{t}^{2}\right\} d z
$$

for any Borel set $E \subset \Omega$. Moreover, if $u_{k} \in C(\Omega)$ are $\mathcal{H}$-convex, and $u_{k} \rightarrow u$ on compact subsets of $\Omega$, then $\mu\left(u_{k}\right)$ converges weakly to $\mu(u)$, that is,

$$
\int_{\Omega} f d \mu\left(u_{k}\right) \rightarrow \int_{\Omega} f d \mu(u)
$$

for any $f \in C(\Omega)$ with compact support in $\Omega$.

Proof. Let $u \in C(\Omega)$ be $\mathcal{H}$-convex, and let $\left\{u_{k}\right\} \subset C^{2}(\Omega)$ be a sequence of $\mathcal{H}$-convex functions converging to $u$ uniformly on compacts of $\Omega$. By Proposition 6.2

$$
\int_{\Omega^{\prime}}\left\{\operatorname{det} \mathcal{H}\left(u_{k}\right)+12\left(\partial_{t} u_{k}\right)^{2}\right\} d z
$$

are uniformly bounded, for every $\Omega^{\prime} \Subset \Omega$, and hence a subsequence of $\left(\operatorname{det} \mathcal{H}\left(u_{k}\right)+\right.$ $\left.12\left(\partial_{t} u_{k}\right)^{2}\right)$ converges weakly in the sense of measures to a Borel measure $\mu(u)$ on $\Omega$. We now prove that the map $u \in C(\Omega) \rightarrow \mu(u) \in M(\Omega)$, the space of finite Borel measures on $\Omega$, is well defined. Accordingly, let $\left\{v_{k}\right\} \subset C^{2}(\Omega)$ be another sequence of $\mathcal{H}$-convex functions converging to $u$ uniformly on compacts of $\Omega$. Assume $\left(\operatorname{det} \mathcal{H}\left(u_{k}\right)+12\left(\partial_{t} u_{k}\right)^{2}\right)$ and $\left(\operatorname{det} \mathcal{H}\left(v_{k}\right)+12\left(\partial_{t} v_{k}\right)^{2}\right)$ converge weakly to Borel measures $\mu, \mu^{\prime}$ respectively. Let $B=B_{R} \Subset \Omega$, and fix $\sigma \in(0,1)$. Let $\eta \in C^{2}(\bar{\Omega})$ be an $\mathcal{H}$-convex function such that $\eta=0$ in $B_{\sigma R}$ and $\eta=1$ on $\partial B_{R} \cdot{ }^{\S}$ From the uniform convergence of $\left\{u_{k}\right\}$ and $\left\{v_{k}\right\}$ towards $u$, given $\varepsilon>0$ there exists $k_{\varepsilon} \in \mathbb{N}$ such that

$$
-\frac{\varepsilon}{2} \leq u_{k}(x)-v_{k}(x) \leq \frac{\varepsilon}{2}, \quad \text { for all } x \in \bar{B} \text { and } k \geq k_{\varepsilon}
$$

Hence

$$
u_{k}+\frac{\varepsilon}{2} \leq v_{k}+\varepsilon \eta
$$

\footnotetext{
${ }^{\S}$ In the $d$-ball $B_{R}(0)$, the function $\eta$ can be constructed as follows. If $v(\xi)=\frac{1}{1-\sigma^{4}}\left(\frac{\|\xi\|^{4}}{R^{4}}-\sigma^{4}\right)$ and $f_{h}$ is the function given by $(6.15)$, then define $\eta(\xi)=f_{h}(v, 0)$ with $h$ sufficiently small.
} 
on $\partial B_{R}$ for $k \geq k_{\varepsilon}$. Define $\Omega_{k}=\left\{\xi \in B_{R}: u_{k}+\frac{\varepsilon}{2}>v_{k}+\varepsilon \eta\right\}$. From Theorem 3.1 we have

$$
\begin{aligned}
\int_{\Omega_{k}}\left\{\operatorname{det} \mathcal{H}\left(u_{k}\right)+12\left(\partial_{t} u_{k}\right)^{2}\right\} d z \leq & \int_{\Omega_{k}} \operatorname{det} \mathcal{H}\left(v_{k}+\varepsilon \eta\right)+12\left(\partial_{t} v_{k}+\varepsilon \partial_{t} \eta\right)^{2} \\
\leq & \int_{B_{R}} \operatorname{det} \mathcal{H}\left(v_{k}\right)+12\left(\partial_{t} v_{k}\right)^{2}+\varepsilon^{2} C \\
& +\varepsilon C \int_{B_{R}}\left(\operatorname{trace} \mathcal{H}_{2}\left(v_{k}\right)+\left|\partial_{t} v_{k}\right|\right) \\
\leq & \int_{B_{R}} \operatorname{det} \mathcal{H}\left(v_{k}\right)+12\left(\partial_{t} v_{k}\right)^{2}+\varepsilon^{2} C \\
& +\varepsilon C \int_{B_{R}}\left(\operatorname{trace} \mathcal{H}_{2}\left(v_{k}\right)+\left|\partial_{t} v_{k}\right|^{2}+1\right)
\end{aligned}
$$

and by Proposition 6.2 and Corollary 6.4 the right hand side is bounded by

$$
\int_{B_{R}} \operatorname{det} \mathcal{H}\left(v_{k}\right)+12\left(\partial_{t} v_{k}\right)^{2}+\varepsilon C .
$$

By definition of $\Omega_{k}$ and since $\eta=0$ in $B_{\sigma R}$, it follows that $B_{\sigma R} \subset \Omega_{k}$ and so by (6.21) we get

$$
\int_{B_{\sigma R}} \operatorname{det} \mathcal{H}\left(u_{k}\right)+12\left(\partial_{t} u_{k}\right)^{2} \leq \int_{B_{R}} \operatorname{det} \mathcal{H}\left(v_{k}\right)+12\left(\partial_{t} v_{k}\right)^{2}+\varepsilon C,
$$

and letting $k \rightarrow \infty$, we get $\mu\left(B_{\sigma R}\right) \leq \mu^{\prime}\left(B_{R}\right)+C \varepsilon$. Hence if $\varepsilon \rightarrow 0$ and $\sigma \rightarrow 1$ we obtain

$$
\mu(B) \leq \mu^{\prime}(B) .
$$

By interchanging $\left\{u_{k}\right\}$ and $\left\{v_{k}\right\}$ we get $\mu=\mu^{\prime}$.

To prove (6.20), we first claim that it holds when $u_{k} \in C^{2}(\Omega)$. Indeed, let $u_{k_{m}}$ be an arbitrary subsequence of $u_{k}$, so $u_{k_{m}} \rightarrow u$ locally uniformly as $m \rightarrow \infty$. By definition of $\mu(u)$, there is a subsequence $u_{k_{m_{j}}}$ such that $\mu\left(u_{k_{m_{j}}}\right) \rightarrow \mu(u)$ weakly as $j \rightarrow \infty$. Therefore, given $f \in C_{0}(\Omega)$, the sequence $\int_{\Omega} f d \mu\left(u_{k}\right)$ and an arbitrary subsequence $\int_{\Omega} f d \mu\left(u_{k_{m}}\right)$, there exists a subsequence $\int_{\Omega} f d \mu\left(u_{k_{m_{j}}}\right)$ converging to $\int_{\Omega} f d \mu(u)$ as $j \rightarrow \infty$ and (6.20) follows. For the general case, given $k$ there exists $u_{j}^{k} \in C^{2}(\Omega)$ such that $u_{j}^{k} \rightarrow u_{k}$ locally uniformly as $j \rightarrow \infty$. By definition of $\mu\left(u_{k}\right)$, there exists a subsequence $u_{j_{m}}^{k}$ such that $\mu\left(u_{j_{m}}^{k}\right) \rightarrow \mu\left(u_{k}\right)$ weakly as $m \rightarrow \infty$. Let $f \in C_{0}(\Omega)$, supp $f=K \subset \Omega^{\prime} \Subset \Omega$. There exists $m_{1}<m_{2}<\cdots$ such that

$$
\left|u_{j_{m_{k}}}^{k}(z)-u_{k}(z)\right|<1 / k, \quad \text { for all } z \in \Omega^{\prime},
$$

and

$$
\left|\int_{\Omega} f d \mu\left(u_{j_{m_{k}}}^{k}\right)-\int_{\Omega} f d \mu\left(u_{k}\right)\right|<1 / k
$$


for $k=1,2, \cdots$. Hence $v_{k}=u_{j_{m_{k}}}^{k} \rightarrow u$ uniformly in $\Omega^{\prime}$ as $k \rightarrow \infty$, and so from the previous claim

$$
\int_{\Omega} f d \mu\left(v_{k}\right) \rightarrow \int_{\Omega} f d \mu(u), \quad \text { as } k \rightarrow \infty
$$

Therefore,

$$
\begin{aligned}
\left|\int_{\Omega} f d \mu\left(u_{k}\right)-\int_{\Omega} f d \mu(u)\right| & \leq\left|\int_{\Omega} f d \mu\left(u_{k}\right)-\int_{\Omega} f d \mu\left(v_{k}\right)\right|+\left|\int_{\Omega} f d \mu\left(v_{k}\right)-\int_{\Omega} f d \mu(u)\right| \\
& \leq \frac{1}{k}+\left|\int_{\Omega} f d \mu\left(v_{k}\right)-\int_{\Omega} f d \mu(u)\right| \rightarrow 0, \text { as } k \rightarrow \infty,
\end{aligned}
$$

and the proof of the theorem is complete.

Corollary 6.6. If $u, v \in C(\bar{\Omega})$ are $\mathcal{H}$-convex in $\Omega, u=v$ on $\partial \Omega$ and $u \geq v$ in $\Omega$, then $\mu(u)(\Omega) \leq \mu(v)(\Omega)$.

\subsection{Comparison principle for $\mathcal{H}$-measures.}

Theorem 6.7. Let $\Omega \subset \mathbb{R}^{3}$ be an open bounded set. If $u, v \in C(\bar{\Omega})$ are $\mathcal{H}$-convex in $\Omega$, $u \leq v$ on $\partial \Omega$ and $\mu(u)(E) \geq \mu(v)(E)$ for each $E \subset \Omega$ Borel set, then $u \leq v$ in $\Omega$.

Proof. Assume $0 \in \Omega, \Delta=\operatorname{diam}(\Omega), \varepsilon>0$, and $u_{\varepsilon}(x, y, t)=u(x, y, t)+\varepsilon\left(x^{2}+y^{2}-\Delta^{2}\right)$. We have $x^{2}+y^{2}-\Delta^{2}<0$ for $(x, y, t) \in \bar{\Omega}$, so $u_{\varepsilon}<u \leq v$ in $\partial \Omega$. Suppose there exists $\left(x_{o}, y_{o}, t_{o}\right) \in \Omega$ such that $u\left(x_{o}, y_{o}, t_{o}\right)>v\left(x_{o}, y_{o}, t_{o}\right)$. Hence the set $D=\{(x, y, t) \in \Omega$ : $\left.u_{\varepsilon}(x, y, t)>v(x, y, t)\right\}$ is non empty for all $\varepsilon$ sufficiently small. In addition, $\bar{D} \cap \partial \Omega=\emptyset$. So $\bar{D} \subset \Omega$ and $u_{\varepsilon}=v$ on $\partial D$. By Corollary 6.6 we get $\mu\left(u_{\varepsilon}\right)(D) \leq \mu(v)(D)$. On the other hand, there exist $u_{k} \in C^{2}(\Omega) \mathcal{H}$-convex in $\Omega$ such that $u_{k} \rightarrow u$ uniformly on compact subsets of $\Omega$. Let $u_{k, \varepsilon}(x, y, t)=u_{k}(x, y, t)+\varepsilon\left(x^{2}+y^{2}-\Delta^{2}\right)$. We have from (4.3) that

$$
\begin{aligned}
\int_{D}\left\{\operatorname{det} \mathcal{H}\left(u_{k, \varepsilon}\right)+\left(u_{k, \varepsilon}\right)_{t}^{2}\right\} d z & =\int_{D}\left\{\operatorname{det} \mathcal{H}\left(u_{k}\right)+2 \varepsilon \operatorname{trace} \mathcal{H}\left(u_{k}\right)+4 \varepsilon^{2}+\left(u_{k}\right)_{t}^{2}\right\} d z \\
& \geq \mu\left(u_{k}\right)(D)+4 \varepsilon^{2}|D| .
\end{aligned}
$$

Letting $k \rightarrow \infty$ we get from Theorem 6.5 that $\mu\left(u_{\varepsilon}\right)(D) \geq \mu(u)(D)+4 \varepsilon^{2}|D|>\mu(u)(D)$ obtaining a contradiction.

\section{REFERENCES}

[1] J. M. Bony. Principe du maximum, inégalité de Harnack et unicitè du problème de Cauchy pour les opérateurs elliptic dégénérés. Ann. Ist. Fourier (Grenoble), 29 (1969) 277-304.

[2] D. Danielli, N. Garofalo, D. M. Nhieu. Notions of convexity in Carnot groups. To appear in Comm. Anal. Geom.

[3] C. E. Gutiérrez. The Monge-Ampère equation. Birkhäuser, Boston, MA, 2001.

[4] G. Lu, J. Manfredi, B. Stroffolini. Convex functions on the Heisenberg group. Preprint.

[5] E. M. Stein. Harmonic Analysis: Real Variable methods, Orthogonality and Oscillatory Integrals Vol. 43 of the Princeton Math. Series. Princeton U. Press. Princeton, NJ, 1993.

[6] N. S. Trudinger, Xu-Jia Wang. Hessian measures I Topol. Methods Nonlinear Anal. 10 (1997) 225239. 
Department of Mathematics, Temple University, Philadelphia, PA 19122

E-mail address: gutierrez@math.temple.edu

Dipartimento di Matemàtica, Università di Bologna, Piazza Porta San Donato 5, Bologna, 40127, ITALY

E-mail address: montanar@dm.unibo.it 